\title{
Results of clubfoot treatment after manipulation and casting using the Ponseti method: experience in Harare, Zimbabwe
}

\author{
Tracey Smythe', Daniel Chandramohan ${ }^{2}$, Jane Bruce ${ }^{2}$, Hannah Kuper', Christopher Lavy ${ }^{3}$ and Allen Foster' \\ 1 International Centre for Evidence in Disability, London School of Hygiene and Tropical Medicine, London, UK \\ 2 London School of Hygiene and Tropical Medicine, London, UK \\ 3 Oxford NIHR Musculoskeletal Biomedical Research Unit, University of Oxford, Headington, UK
}

Abstract

OBjectives The objective of this study was to evaluate the outcomes of the Ponseti manipulation and casting method for clubfoot in a tertiary hospital in Zimbabwe and explore predictors of these outcomes.

METHODs A cohort study included children with idiopathic clubfoot managed from 2011 to 2013 at Parirenyatwa Hospital. Demographic data, clinical features and treatment outcomes were extracted from clinic records. The primary outcome measure was the final Pirani score (clubfoot severity measure) after manipulation and casting. Secondary outcomes included change in Pirani score (pretreatment to end of casting), number of casts for correction, proportion receiving tenotomy and proportion lost to follow up.

RESUlTs A total of 218 children (337 feet) were eligible for inclusion. The median age at treatment was 8 months; 173 children (268 feet) completed casting treatment within the study period. The mean length of time for corrective treatment was 10.2 weeks (9.5-10.9 weeks). Of the 45 children who did not complete treatment, 28 were under treatment and 17 were lost to follow up. A Pirani score of 1 or less was achieved in $85 \%$ of feet. Mean Pirani score at presentation was 3.80 (SD 1.15) and post-treatment 0.80 (SD 0.56, $P$-value $<0.0001$ ). Severity of deformity and being male were associated with a higher (worse) final Pirani score. Severity and age over two were associated with an increase in the number of casts required to correct deformity.

CONCLUSION This case series demonstrates that the majority $(80 \%+)$ of children with clubfoot can achieve a good outcome with the Ponseti manipulation and casting method.

keywords clubfoot, congenital talipes equinovarus, Zimbabwe, Ponseti, treatment, cohort analysis

\section{Introduction}

Congenital talipes equinovarus (CTEV), a deformity commonly called clubfoot, is a complex congenital condition affecting the foot. It presents with varying degrees of rigidity that is not passively correctable [1]. Untreated clubfoot results in pain and disability [2]. The incidence of clubfoot varies globally [3] and it is estimated that $80 \%$ of children born with clubfoot each year live in low- and middle-income countries (LMICs) [4]. Boys are affected 2.5 times as often as girls, and the condition is bilateral in half of the cases [5]. The causes of clubfoot are poorly understood, but may include genetic factors [6].

The Ponseti method of conservative clubfoot management is considered the best practice [7] and avoids corrective surgery in over $90 \%$ of cases [8]. Use of a primarily non-operative technique is beneficial in LMICs where there are limited resources and different cadres of health workers can be trained to treat clubfoot [9].

The Ponseti method is divided into two distinct phases: the corrective phase and the maintenance phase. The corrective phase involves manipulation of the foot followed by casting with plaster of paris. The cast holds the stretch achieved through manipulation of tight structures and allows time for soft tissue remodelling and correction of the position of the bones in the foot [10]. Sequential correction of the cavus, adductus and varus of clubfoot occurs around the talus [8]. A percutaneous Achilles tenotomy is usually required to correct the residual equinus (downward position of the foot), followed by 3 weeks in a cast to assist healing [11].

The maintenance phase consists of a bracing regime to prevent recurrence. The foot abduction brace is worn $23 \mathrm{~h}$ a day for the first 3 months and then at night during sleep until five years of age [11]. 
T. Smythe et al. Clubfoot treatment after manipulation and casting using the Ponseti method

This study examines the outcomes of the corrective phase (manipulation and casting) for clubfoot as recommended by the Ponseti method in a cohort of children undergoing treatment at one referral hospital in Zimbabwe.

\section{Methods}

Ethical approval for this study was granted by the Joint Research Ethics Committee for the University of Zimbabwe, College of Health Sciences and Parirenyatwa Group of Hospitals, and the ethics committees at the Medical Research Council of Zimbabwe and the London School of Hygiene and Tropical Medicine.

\section{Study design}

A cohort included children being treated for idiopathic clubfoot at Parirenyatwa Hospital's clubfoot outpatient clinic in Harare from 22 March 2011 to 23 April 2013 (25 months). All children with a diagnosis of unilateral or bilateral idiopathic clubfoot corrected by the Ponseti method at the study hospital were eligible. There was no restriction on previous treatment for clubfoot. The only exclusion criterion was foot conditions other than idiopathic clubfoot, for example clubfoot associated with a syndrome or comorbidity, such as spina bifida.

Children aged under 2 years. The corrective phase of the Ponseti method followed the kinematic principles of correction [10] and involved weekly cast changes. The knee was fixed at 90 degrees in the long leg plaster of paris casts (toe to groin). Physiotherapists performed the manipulation and casting, and casts were removed by parents at the clinic in the morning of the appointment. Medical officers performed a percutaneous Achilles tenotomy with local anaesthetic when indicated by clinical assessment. The foot was held in a long leg plaster cast in abduction and dorsiflexion for 3 weeks post-tenotomy.

The maintenance phase used the Steenbeek foot abduction brace [12] to hold both feet in seventy degrees abduction. The brace was made locally and supply was regular. The brace regime was initiated on the day of removal of the final cast.

Children aged over 2 years. Children over 2 years were treated with the modified principles of the Ponseti method as recommended for older children [13]. Abduction of thirty to forty degrees was achieved with manipulation and long leg plaster casts with seventy degrees of knee flexion. The casts were changed weekly. A percutaneous Achilles tenotomy was performed for all children aged over 2 years with residual equinus and the cast post-tenotomy remained for 4-6 weeks. In the maintenance phase, the foot abduction brace held both feet in thirty degrees of abduction.

The key variables recorded on the clinic records by physiotherapists and extracted by the researchers, included child's date of birth, gender, diagnosis, order of birth, mother's date of birth, place of birth, family history of clubfoot, side of affected foot, history of previous treatment and weekly recording of Pirani score. The Pirani score is a classification system for assessing severity of clubfoot based on clinical examination [14] and was scored by the clinic physiotherapists. A decrease in score correlates to improvement in deformity. The Pirani score is from 0 to 6 where zero is a normal foot and six is the most severe deformity. Scoring is performed at half-point intervals.

\section{Data management and analysis}

Data were managed and analysed using Stata 14.1, StataCorp 4905, Lakeway Drive College Station, Texas 77845, USA. A hierarchical conceptual framework for analysis was developed [15]. The primary outcome measure was defined as Pirani score at the end of the manipulation and casting phase, with success determined as a score of 1 or less. The following secondary outcomes were defined: (i) the change in Pirani score from pre-treatment (first manipulation and casting appointment) to post-treatment (first brace fitting); (ii) number of casts required for correction, inclusive of the final cast after tenotomy; (iii) proportion having a tenotomy; (iv) proportion lost to follow up (defaulted appointment during casting and was not seen again).

Three factors that may influence the Ponseti manipulation and casting process were identified a priori: age at first treatment, history of previous treatment and pretreatment severity of deformity. Age at first treatment and a combination of treatments may affect the extent of clubfoot correction [16]. Response to casting may be influenced by initial presenting severity [4, 17]. Gender was included as a fourth possible predictor according to the hierarchical conceptual framework.

Age at first treatment was defined as a binary variable ( $\leq 2$ or $>2$ years) to allow comparison to findings from the literature. An ordered categorical variable for pre-treatment severity of deformity was created based on Pirani score before treatment. An increase of 1 in the categorical variable represents an increase of 0.5 in the Pirani score.

\section{Statistical methods}

The associations between outcomes and gender, age at first treatment, history of previous treatment and severity 
T. Smythe et al. Clubfoot treatment after manipulation and casting using the Ponseti method

category were explored. Statistical significance for the case series was set at the $95 \%$ confidence level.

The outcomes of Pirani score (final and change in score) were analysed by feet. The outcomes of number of casts, tenotomy performed and loss to follow up were analysed by child.

Unilateral and bilateral clubfeet were included in analysis of the Pirani score. As right and left feet are correlated in the same child [18], a random effects model was used to assess the association between potential predictors and the Pirani score. The random effects model means that the right and left feet on the same child were not treated as independent. A linear regression model was fitted for the outcome of number of casts. This was possible on an individual level as children with bilateral clubfeet had the same number of casts applied per foot to enable both tenotomies to be performed on the same day. A logistic regression model was fitted to assess factors associated with the binary variables of tenotomy performed and loss to follow up. The models assumed no effect modification.

A second model was constructed with a backward elimination approach to identify a model of best fit and the joint predictive capability of the variables [19]. Any variable that affected the primary outcome by less than $10 \%$ was dropped and not considered as a confounder [19]. Further adjustment through backward elimination did not change the outcome of the first random effects model and is not included in the results.

\section{Results}

\section{Characteristics}

A total of 218 children (337 feet) with a diagnosis of idiopathic clubfoot presented for treatment (Figure 1); 28 children were excluded from analysis as they had yet to

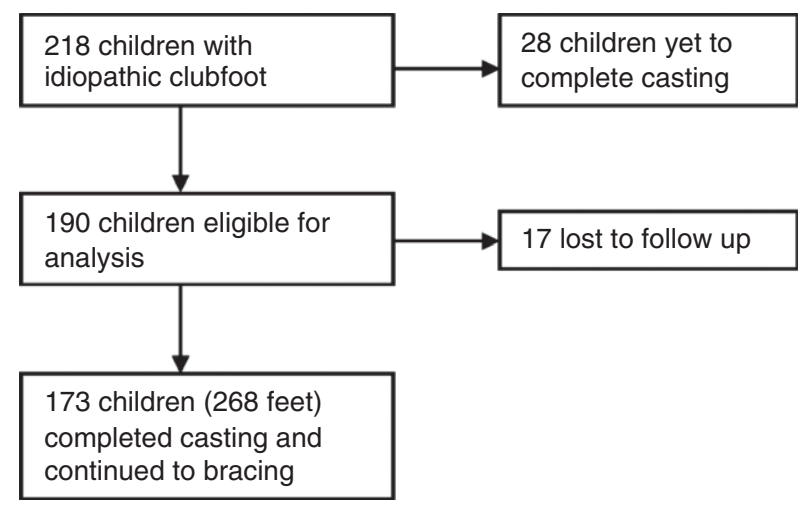

Figure I Flow chart of clubfoot treatment pathway. complete the manipulation and casting treatment, and of the remaining 190 children, 17 (8.9\%) were lost to follow up during the casting period. Loss to follow up was not associated with gender, age at first treatment, previous treatment or severity; 173 children completed treatment within the study time period and continued to the bracing phase (Table 1). Mean length of time for the corrective phase was 10.2 weeks (9.5-10.9 weeks) and is inclusive of the final cast post-tenotomy.

Bilateral clubfoot was seen in 90 children (52\%); 83 (48\%) had unilateral clubfoot (37 left and 46 right). The sample included more males than females. The median age at first treatment was 8 months (interquartile range 2.8 months to 20.1 months old); 169 (97.7\%) children

Table I Characteristics of the children who completed manipulation and casting $(N=173)$

\begin{tabular}{lr}
\hline Characteristics & $N(\%)^{*}$ \\
\hline Gender & \\
Male & $119(68.8)$ \\
Female & $54(31.2)$ \\
Previous treatment $\dagger$ & \\
$\quad$ None & $40(23.7)$ \\
Kite Method & $118(69.8)$ \\
Long leg cast & $8(4.7)$ \\
Short leg cast and surgery & $2(1.2)$ \\
Strapping & $1(0.6)$ \\
Presentation age & \\
$\leq 2$ years & $143(82.7)$ \\
$>2$ years & $30(17.3)$ \\
Birthplace $\$$ & \\
Hospital & $73(45.3)$ \\
Clinic & $73(45.3)$ \\
Home & $15(9.2)$ \\
Side of clubfoot & \\
Bilateral & $90(52.0)$ \\
Unilateral & $83(48.0)$ \\
Birth order $\dagger$ & \\
First born & $61(36.1)$ \\
Second born & $47(27.8)$ \\
Third born & $37(21.9)$ \\
$\geq$ Fourth born & $24(14.2)$ \\
Previous family history $\$$ & $18(10.5)$ \\
Yes & $153(89.5)$ \\
No & $23(14.3)$ \\
Age of mother at first treatment $\$$ & $89(55.3)$ \\
$\leq 20$ & $42(26.1)$ \\
$20.1-30$ & $7(4.3)$ \\
30.1-40 & \\
$\geq 40.1$ & \\
\hline
\end{tabular}

*Percentage excluding missing values.

${ }^{\dagger}$ Missing for 4 children $(2.3 \%)$.

${ }^{*}$ Missing for 12 children $(6.9 \%)$.

${ }^{\S}$ Missing for 2 children $(1.2 \%)$. 


\section{T. Smythe et al. Clubfoot treatment after manipulation and casting using the Ponseti method}

were under the age of 5 years, with four children between 5 and 6 years of age. At presentation, 67 $(38.7 \%)$ children were under 6 months, and $40(23.7 \%)$ children were new referrals with no previous treatment; 118 of $129(91.5 \%)$ children with a history of previous treatment were treated with below-knee casts using the Kite method [20]. The mean number of casts reported with the Kite method was 16.03 (95\% CI: 13.94-18.12). Less than $10 \%$ of children were born at home. A family history of clubfoot was reported by $18(10.5 \%)$ parents.

\section{Pirani score}

Of the 173 children (268 feet) who completed casting, 161 children (246 feet) had a full record of pre- and post-treatment Pirani scores (Table 2).

At the start of treatment, 221 of 246 feet $(89.8 \%$ boys $90.9 \%$ and girls $89.2 \%$ ) had a Pirani score of 2.0 or more. After completion of the corrective phase, 209 of 246 feet $(85.0 \%)$ achieved a Pirani score of 1 or less, of which 37 feet had a Pirani score of 0; 99 achieved 0.5; and 73 had a Pirani score of 1 . A further $27(11.0 \%)$ feet had a Pirani score of 1.5 and $10(4.0 \%)$ failed to achieve a score of 1.5 or less.

Table 2 Pre- and post-treatment Pirani scores of feet that completed casting

\begin{tabular}{lcc}
\hline & $\begin{array}{l}\text { Pre-treatment foot } \\
\text { Pirani score }\end{array}$ & $\begin{array}{l}\text { Post-treatment foot } \\
N^{*}(\%) \dagger\end{array}$ \\
\hline $0-1.0$ & $13(5.3)$ & $209(85.0)$ \\
1.5 & $12(4.9)$ & $27(11.0)$ \\
$2.0-6.0$ & $221(89.8)$ & $10(4.0)$ \\
Total & $246(100.0)$ & $246(100.0)$ \\
\hline
\end{tabular}

*Missing for 22 children.

†Percentage excluding missing values.
A Pirani score of 1 or less was achieved more frequently in girls with clubfoot than boys $(94.0 \% \mathrm{vs}$. $81.5 \%$ ). The multivariable adjusted regression model showed that a girl's foot was 7.49 (95\% CI: 1.24-45.45) times more likely to achieve a score of 1 or less after manipulation and casting than that of a boy. A more severe clubfoot was less likely to achieve an end Pirani score of 1 or less $(\mathrm{AOR}=0.75,95 \% \mathrm{CI}$ : 0.56-0.99). Age at first treatment and previous treatment were not associated with final Pirani score (Table 3).

The mean Pirani score on presentation was 3.80 (SD $1.15)$, and mean Pirani score on final cast removal was 0.80 (SD 0.56) with $P<0.001$ on paired t-test for change in Pirani score. Change in Pirani score is demonstrated in Figure 2.

Boys with clubfoot experienced less change in score than girls [ -0.25 (95\% CI: -0.43 to -0.07$)$ (Table 4$)$. A more severe clubfoot was likely to have a greater change in score with treatment. There is no evidence that age at first treatment or history of previous treatment is associated with change in Pirani score after multivariable adjustment.

The mean initial Pirani score for a girl's foot was 3.40 (95\% CI: 3.05-3.74) and for a boy's foot, 3.76 (95\% CI: 3.60-3.93). While there was overlap in the confidence intervals, there is a trend for girls having less severe deformity at baseline, which may explain the better (lower) final Pirani score outcome.

\section{Number of casts}

The mean number of casts required in the case series to completion of corrective phase was 7.27 (95\% CI 6.77.9) (Table 5). Children under the age of 2 years required fewer casts $(-1.72,95 \% \mathrm{CI}-3.27$ to -0.18$)$, but the more severe the initial deformity, the more casts were required $(0.75,95 \% \mathrm{CI}: 0.44-1.06)$. There was no

Table 3 Factors associated with a Pirani score of 1 or less (Number of feet $=246)$

\begin{tabular}{|c|c|c|c|c|c|}
\hline Predictor Variable & Category & $\begin{array}{l}\text { Score } \leq 1 \\
\text { Feet } N(\%)\end{array}$ & $\begin{array}{l}\text { Score }>1 \\
\text { Feet } N(\%)\end{array}$ & Crude OR $(95 \%, \mathrm{CI})$ & $\begin{array}{l}\text { AOR for all variables* } \\
(95 \%, \mathrm{CI})\end{array}$ \\
\hline \multirow[t]{2}{*}{ Gender } & Male & $146(81.6)$ & $33(18.4)$ & 1 & 1 \\
\hline & Female & $63(94.0)$ & $4(6.0)$ & $5.13(1.07,24.70)$ & $7.49(1.24,45.45)$ \\
\hline \multirow[t]{2}{*}{ Age at first treatment } & $>2$ years & $31(70.5)$ & $13(29.5)$ & 1 & 1 \\
\hline & $\leq 2$ years & $178(88.1)$ & $24(11.9)$ & $4.89(1.23,19.49)$ & $1.13(0.31,4.10)$ \\
\hline \multirow{2}{*}{ Previous treatment } & No & $52(91.2)$ & $5(8.8)$ & 1 & 1 \\
\hline & Yes & $154(83.7)$ & $30(16.3)$ & $0.43(0.11,1.69)$ & $0.46(0.11,1.95)$ \\
\hline Pre-treatment severity category & Unit incre & & & $0.82(0.63,1.06)$ & $0.75(0.56,0.99)$ \\
\hline
\end{tabular}

*Adjusted for place of birth, family history, birth order, affected foot, mother's age and remaining three potential predictors using a random effects model. 
T. Smythe et al. Clubfoot treatment after manipulation and casting using the Ponseti method

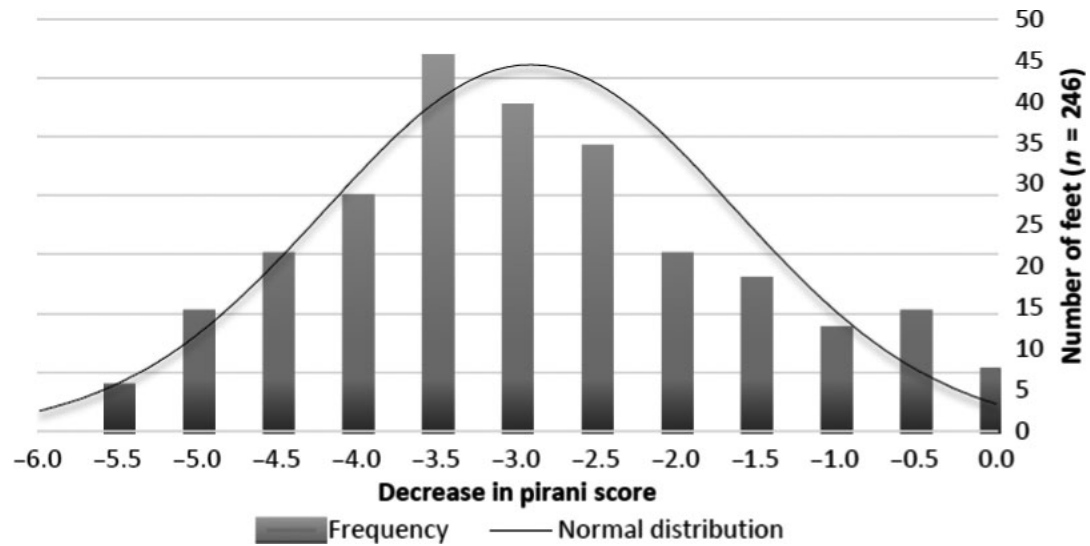

Figure 2 Distribution of change in Piran score from pre- to post-treatment.

Frequency Normal distribution

Table 4 Predictors of change in pre- to post-treatment Pirani scores $(N$ feet $=246)$

\begin{tabular}{lllll}
\hline Predictor variable & Category & $\begin{array}{l}\text { Mean score change } \\
(95 \%, \mathrm{CI})\end{array}$ & $\begin{array}{l}\text { Crude coefficient } \\
(95 \%, \mathrm{CI})\end{array}$ & $\begin{array}{l}\text { Adjusted coefficient* } \\
(95 \%, \mathrm{CI})\end{array}$ \\
\hline Gender & Female & $2.87(2.52,3.23)$ & Reference & $\begin{array}{l}\text { Reference } \\
-0.25(-0.43,-0.07)\end{array}$ \\
Age at first treatment & Male & $2.93(2.75,3.11)$ & $0.07(-0.35,0.50)$ & $\begin{array}{l}\text { Reference } \\
\text { Reference }\end{array}$ \\
Previous treatment & $>2$ years & $2.59(2.23,2.96)$ & $0.50(-0.01,1.01)$ & $-0.01(-0.23,0.21)$ \\
& $\leq 2$ years & $2.99(2.81,3.17)$ & Reference & $0.06(-0.13,0.26)$ \\
Pre-treatment severity category & No & $3.57(3.25,3.89)$ & $-0.91(-1.35,-0.47)$ & $0.47(0.44,0.50)$ \\
\hline
\end{tabular}

*Adjusted for place of birth, family history, birth order, affected foot, mother's age and remaining three potential predictors using a random effects model.

Table 5 Factors associated with number of casts $(N=164)$

\begin{tabular}{|c|c|c|c|c|}
\hline Predictor Variable & Category & $\begin{array}{l}\text { Mean number of casts } \\
(95 \%, \mathrm{CI})\end{array}$ & $\begin{array}{l}\text { Crude coefficient } \\
(95 \%, \mathrm{CI})\end{array}$ & $\begin{array}{l}\text { Adjusted coefficient* } \\
(95 \%, \mathrm{CI})\end{array}$ \\
\hline \multirow[t]{2}{*}{ Gender } & Female & $6.53(5.47,7.58)$ & Reference & Reference \\
\hline & Male & $7.61(6.80,8.42)$ & $0.65(-0.69,2.00)$ & $0.53(-0.87,1.94)$ \\
\hline \multirow[t]{2}{*}{ Age at first treatment } & $>2$ years & $9.04(7.25,10.83)$ & Reference & Reference \\
\hline & $\leq 2$ years & $6.90(6.22,7.57)$ & $-1.72(-3.27,-0.18)$ & $-2.49(-4.10,-0.88)$ \\
\hline \multirow[t]{2}{*}{ Previous Treatment } & No & $7.55(6.26,8.84)$ & Reference & Reference \\
\hline & Yes & $7.24(6.46,8.01)$ & $-0.62(-2.10,0.85)$ & $-0.37(-1.16,1.90)$ \\
\hline Pre-treatment severity category & Unit increase & & $0.75(0.48,1.01)$ & $0.75(0.44,1.06)$ \\
\hline
\end{tabular}

*Adjusted for place of birth, family history, birth order, affected foot, mother's age and remaining three potential predictors.

association between gender or previous treatment and the number of casts required to achieve correction.

\section{Achilles tenotomy}

A percutaneous Achilles tenotomy was performed on 127 $(78.9 \%)$ of 161 children who completed treatment to bracing phase; 69 tenotomies were on bilateral feet and 58 on unilateral (Table 6).

A child with a history of previous treatment was 8.15 (95\% CI: 2.00-33.20) times more likely to have a tenotomy performed than a child with no prior treatment. An increase of pre-treatment Pirani score increased the likelihood that a tenotomy was performed $(\mathrm{AOR}=1.72$, 95\% 
T. Smythe et al. Clubfoot treatment after manipulation and casting using the Ponseti method

Table 6 Factors associated with tenotomy $(N=173)$

\begin{tabular}{|c|c|c|c|c|c|}
\hline Predictor variable & Category & $\begin{array}{l}\text { Tenotomy } \\
N(\%)\end{array}$ & $\begin{array}{l}\text { No Tenotomy } \\
N(\%)\end{array}$ & Crude OR $(95 \%, \mathrm{CI})$ & Adjusted OR* $(95 \%, \mathrm{CI})$ \\
\hline \multirow[t]{2}{*}{ Gender } & Female & $36(72.0)$ & $14(28.0)$ & 1 & \\
\hline & Male & $91(81.3)$ & $21(18.7)$ & $1.69(0.77,3.67)$ & $1.72(0.54,5.48)$ \\
\hline \multirow[t]{2}{*}{ Age at first treatment } & $>2$ years & $23(79.3)$ & $6(20.7)$ & 1 & \\
\hline & $\leq 2$ years & $104(78.2)$ & $29(21.8)$ & $0.94(0.35,2.51)$ & $0.99(0.20,4.99)$ \\
\hline \multirow[t]{2}{*}{ Previous treatment } & No & $27(69.2)$ & $12(30.8)$ & 1 & \\
\hline & Yes & $97(80.2)$ & $24(19.8)$ & $1.64(0.72,3.78)$ & $8.15(2.00,33.20)$ \\
\hline Pre-treatment severity category & Unit increa & & & $1.51(1.26,1.81)$ & $1.72(1.33,2.23)$ \\
\hline
\end{tabular}

*Adjusted for place of birth, family history, birth order, affected foot, mother's age and remaining three potential predictors.

CI: 1.33-2.23). There was no evidence for an association between gender or age at first treatment and whether a tenotomy was performed.

\section{Discussion}

The gender ratio and bilateral to unilateral clubfoot ratio are similar to global figures, with more males than females (2.2:1) and slightly more bilateral than unilateral cases [21]. The most common presenting Pirani (severity) score was 4.5 , suggestive of a moderately severe clubfoot presentation, although less severe than other clubfoot cohort reports in the region $[17,22,23]$. Children attended Parirenyatwa clubfoot clinic prior to the introduction of the Ponseti method when treatment was with the Kite method [20]. The reduced initial Pirani scores are likely to be due to the inclusion of 118 children who had previous treatment with the Kite method. The mean age at first treatment was 13.5 months and less than $39 \%$ of children presented under 6 months of age.

This large cohort in Zimbabwe found that the Ponseti manipulation and casting method was successful in $85 \%$ of feet, when success is defined as a Pirani score of 1 or less at the end of the correction phase. However, further follow-up of this cohort is required to evaluate the longer-term outcome after bracing up to the age of 5 years. It will be important to see whether the good results obtained in the manipulation and casting phase can be maintained through the bracing phase when compliance with treatment is likely to be a greater problem. Also, it will be necessary to evaluate the patient or carer reported functional outcome, as well as the anatomical structural outcomes [24].

The only predictor of low Pirani score at the end of casting was female gender. Severity of deformity was associated with a larger change in Pirani score (pre- to post-treatment), which is in line with current literature that suggests the Ponseti method is also effective in severe cases [25]; however, a higher Pirani score at baseline was less likely to achieve a satisfactory outcome (Pirani score of 1 or less) on completion of manipulation and casting.

Children with high Pirani scores at presentation (severity of clubfoot) were more likely to require additional casts as expected $[17,25,26]$. In addition, more casts were required for the correction of clubfoot for children over 2 years.

The proportion of children having tenotomy in the current series $(78.9 \%$ of children $/ 79.7 \%$ of clubfeet) is higher than reported in most studies from sub-Saharan Africa [27]. The Parirenyatwa clubfoot clinic is a physiotherapist-led clinic and communication with the orthopaedic department occurred regularly regarding the progress of correction and timing of tenotomy. Medical officers and equipment were available to complete the required tenotomies upon request.

Children with high Pirani scores at presentation were more likely to require tenotomy, as shown previously [28]. This is likely due to tighter connective tissues which are evident in severe deformity and could relate to a biomechanical difference in the collagen itself [29]. In addition, children with a history of previous treatment were over eight times more likely to have a tenotomy performed than if there was no history of prior treatment. This may be due to previous inadequate management with the Kite procedure [30], or because children referred to Parirenyatwa with a history of previous treatment may have had more severe deformity, as children treated successfully elsewhere would be less likely to present to the clinic.

Seventeen children were lost to follow up during casting. No attempt was made to contact those lost to follow up. While services are provided free of charge for children under the age of five at Parirenyatwa, further cultural, financial and structural barriers pertinent to surgery [31] may contribute to the ability to adhere to treatment. 
T. Smythe et al. Clubfoot treatment after manipulation and casting using the Ponseti method

Results of the current cohort of children treated at Parirenyatwa hospital may not be representative of other settings in Zimbabwe. This tertiary referral clubfoot clinic had a regular supply of plaster of paris, Steenbeek foot abduction braces and access to Medical Officers during the study time period. It is difficult to generalise the results to clinics that experience shortages of orthopaedic, surgical and material resources.

Well-maintained medical records for a large cohort of children in a low-income country are strengths of this study and the 173 cases provide one of the largest case series reported in sub-Saharan Africa.

The study has a number of limitations. There was no comparator (control or other treatment group) within the case series. Although attribution of the change in Pirani score to the intervention is plausible, it is not proven. The study participants were self-selected and therefore selection bias cannot be ruled out. In addition, the Pirani score has been reported to have good intraand inter-rater reliability and validity in young children [14] and is recommended for use in LMICs [32]; however, it is not validated for older children. No information was available from the medical records as to whether further treatment including surgery was performed on the 37 feet that failed to achieve a Pirani score of 1.

Plausible evidence from case studies, before-and-after treatment studies and a small number of RCTs suggests the minimally invasive Ponseti method as the best practice. Continued evaluation of treatment results is required, and in the light of the evidence, randomised controlled trials with historical techniques are unethical. A standardised method of reporting service delivery is necessary for the evaluation of outcomes between clinics and regions. In addition, clearly outlined protocols will assist further exploration of the Ponseti method outcomes and predictors of success for clubfoot management.

In conclusion, a consensus is required on a simple userfriendly definition of success for the Ponseti method to compare one series with another and to assist field therapists monitor the results. Success for this case series on the completion of the corrective phase of treatment was defined as a Pirani score of 1 or less. The case series demonstrates that the majority $(80 \%+)$ of children with clubfoot can achieve a Pirani score of 1 or less, with a relatively small loss to follow up $(8.9 \%)$ to completion of corrective phase. Age at presentation and previous history of treatment did not greatly affect the final Pirani score. Follow-up is required to assess maintenance of a good result and if a low dropout rate can be maintained through bracing.

\section{Acknowledgements}

We thank the Zimbabwe Ministry of Health and Child Care, the Parirenyatwa Clubfoot Clinic Staff, Dr Max Gova and his orthopaedic team, Mrs R Muzarurwi, Ms C Ncube and the physiotherapy team, Ryan Bathurst and Debra Mudariki of The Zimbabwe Sustainable Clubfoot Programme, for their diligence in the treatment of clubfoot and support of this project. The Beit Trust is gratefully acknowledged for the scholarship awarded to TS.

\section{References}

1. Bridgens J, Kiely N. Current management of clubfoot (congenital talipes equinovarus). BMJ 2010: 340: c355.

2. Pirani S, Naddumba E, Mathias R et al. Towards effective ponseti clubfoot care: the Uganda sustainable clubfoot care project. Clin Orthop Relat Res 2009: 467: 1154-1163.

3. Carroll NC. Clubfoot in the twentieth century: where we were and where we may be going in the twenty-first century. J Pediatr Orthop B 2012: 21: 1-6.

4. Jowett CR, Morcuende JA, Ramachandran M. Management of congenital talipes equinovarus using the Ponseti method: a systematic review. J Bone Joint Surg 2011: 93-B: 11601164.

5. Gibbons PJ, Gray K. Update on clubfoot. J Paediatr Child Health 2013: 49: E434-E437.

6. Siapkara A, Duncan R. Congenital talipes equinovarus: a review of current management. J Bone Joint Surg 2007: 89B: $995-1000$.

7. Cooper DM, Dietz FR. Treatment of idiopathic clubfoot. A thirty-year follow-up note. J Bone Joint Surg Am 1995: 77: 1477-1489.

8. Ponseti IV. The treatment of congenital clubfoot. J Orthop Sports Phys Ther 1994: 20: 1.

9. Owen RM, Kembhavi G. A critical review of interventions for clubfoot in low and middle-income countries: Effectiveness and contextual influences. J Pediatr Orthop B 2012: 21: 59-67.

10. Ponseti IV, Campos J. Observations on pathogenesis and treatment of congenital clubfoot. Clin Orthop Relat Res 1972: 84: 50-60.

11. Ponseti IV, Campos J. The classic: observations on pathogenesis and treatment of congenital clubfoot. Clin Orthop Relat Res 2009: 467: 1124-1132.

12. Mang'oli P, Theuri J, Kollmann T, MacDonald NE. Ponseti clubfoot management: experience with the Steenbeek foot abduction brace. Paediatr Child Health 2014: 19: 513-514.

13. Lourenco AF, Morcuende JA. Correction of neglected idiopathic club foot by the Ponseti method. J Bone Joint Surg Br 2007: 89: 378-381.

14. Pirani S, Hodges D, Sekeramayi F. A relaible and valid method of assessing the amount of deformity in the congenital clubfoot deformity. J Bone Joint Surg 2008: 90-B (Suppl. I): 53. 
T. Smythe et al. Clubfoot treatment after manipulation and casting using the Ponseti method

15. Victora CG, Huttly SR, Fuchs SC, Olinto MT. The role of conceptual frameworks in epidemiological analysis: a hierarchical approach. Int J Epidemiol 1997: 26: 224-227.

16. Zhao D, Li H, Zhao L, Liu J, Wu Z, Jin F. Results of clubfoot management using the Ponseti method: do the details matter? A systematic review. Clin Orthop Relat Res 2014: 472: 1329-1336.

17. Lavy C, Mannion S, Mkandawire $\mathrm{N}$ et al. Club foot treatment in Malawi: a public health approach. Disabil Rehabil 2007: 29: 857-862.

18. Gray K, Gibbons P, Little D, Burns J. Bilateral clubfeet are highly correlated: a cautionary tale for researchers. Clin Orthop Relat Res 2014: 472: 3517-3522.

19. Kirkwood B, Sterne J. Essential Medical Statistics (2nd edn), Blackwell: Oxford, 2003.

20. Kite JH. Nonoperative treatment of congenital clubfoot. Clin Orthop Relat Res 1972: 84: 29-38.

21. Bass A. Update on club foot. Paediatr Child Health 2012: 22: 239-242.

22. Ayana B, Klungsoyr PJ. Good results after Ponseti treatment for neglected congenital clubfoot in Ethiopia. A prospective study of 22 children (32 feet) from 2 to 10 years of age. Acta Orthopaedica 2014: 85: 641-645.

23. Harnett P, Freeman R, Harrison WJ, Brown LC, Beckles V. An accelerated Ponseti versus the standard Ponseti method: a prospective randomised controlled trial. J Bone Joint Surg 2011: 93-B: 404-408.

24. Perveen R, Evans AM, Ford-Powell V et al. The Bangladesh clubfoot project: audit of 2-year outcomes of Ponseti treatment in 400 children. J Pediatr Orthop 2014: 34 :

720-725.

25. Zhang W, Richards BS, Faulks ST, Karol LA, Rathjen KA, Browne RH. Initial severity rating of idiopathic clubfeet is an outcome predictor at age two years. J Pediatr Orthop B 2012: 21: 16-19.

26. Dyer PJ, Davis N. The role of the Pirani scoring system in the management of club foot by the Ponseti method. J Bone Joint Surg Br 2006: 88: 1082-1084.

27. Harmer L, Rhatigan J. Clubfoot care in low-income and middle-income countries: from clinical innovation to a public health program. World J Surg 2014: 38: 839-848.

28. Scher DM, Feldman DS, van Bosse HJ, Sala DA, Lehman WB. Predicting the need for tenotomy in the Ponseti method for correction of clubfeet. J Pediatr Orthop 2004: 24: 349-352.

29. Krauspe R, Wess KM, Raab P, Stahl U, Ronneberger D, Fietzek PP. Biomechanical analysis of collagen in clubfeet and in controls. J Bone Joint Surg 2002: 84-B (Suppl. I): 17 -c-.

30. Rijal R, Shrestha BP, Singh GK et al. Comparison of Ponseti and Kite's method of treatment for idiopathic clubfoot. Indian J Orthop 2010: 44: 202-207.

31. Grimes CE, Bowman KG, Dodgion CM, Lavy CB. Systematic review of barriers to surgical care in low-income and middle-income countries. World J Surg 2011: 35: 941-950.

32. Staheli L. Clubfoot: Ponseti Method. Global Help, 2009. (Available from: http://www.global-help.org/publications/ books/help_cfponseti.pdf) [01 May 2016].

Corresponding Author Tracey Smythe, International Centre for Evidence in Disability, London School of Hygiene and Tropical Medicine, Keppel Street, London WC1E7HT, UK. Tel: +44 2079 588348; E-mail: tracey.smythe@lshtm.ac.uk 